\title{
Berufsgruppen- und altersabhängige Unterschiede in der Arbeitsunfähigkeit durch Varizen der unteren Extremitäten
}

\section{Age- and occupation-dependent differences in sick leave due to varicose veins in the lower extremities}

\author{
Autoren \\ Institute \\ 1 Bundesanstalt für Arbeitsschutz und Arbeitsmedizin, \\ Berlin \\ 2 Charité - Universitätsmedizin Berlin, corporate member \\ of Freie Universität Berlin, Humboldt-Universität zu \\ Berlin, and Berlin Institute of Health \\ 3 Bayerisches Krebsregister, Landesamt für Gesundheit und \\ Lebensmittelsicherheit
}

Claudia Brendler ${ }^{1,2}$, Falk Liebers ${ }^{1}$, Jacqueline Müller-Nordhorn ${ }^{2,3}$, Ute Latza ${ }^{1,2}$

\section{Schlüsselwörter}

Varizen, Beruf, Arbeitsunfähigkeit, Alter

Key words

varicosse veins, occupation, sick leave, age

eingereicht $\quad 08.05 .2018$

akzeptiert $\quad 02.08 .2018$

Bibliografie

DOI https://doi.org/10.1055/a-0852-0088

Phlebologie 2019; 48: 103-111

(c) Georg Thieme Verlag KG Stuttgart · New York

ISSN 0939-978X

Korrespondenzadresse

Claudia Brendler, MPH

Bundesanstalt für Arbeitsschutz und Arbeitsmedizin (BAuA)

Nöldnerstraße 40/42

10317 Berlin

Tel.: +4930515484230

Email: brendler.claudia@baua.bund.de

Englische Version unter:

https://doi.org/10.1055/a-0852-0088

\section{ZUSAMMENFASSUNG}

Hintergrund/Zielsetzung Arbeitsunfähigkeit (AU) durch Venenerkrankungen ist relativ häufig. Sie ist bei Erwerbstätigen abhängig von der beruflichen Tätigkeit. Es soll geklärt werden, ob berufsspezifische Unterschiede im Auftreten von AU-Ereignissen aufgrund der ICD 10-Diagnose „Varizen der unteren Extremitäten“ zudem abhängig vom Lebensalter sind.

Methodik Die Untersuchung basiert auf einer Sekundärdatenanalyse von AU-Daten fast aller gesetzlichen Krankenkassen
Deutschlands des Jahres 2008. Es liegen geschlechtsstratifizierte, aggregierte Daten von 26,2 Mio. gesetzlich pflichtversicherten Erwerbstätigen vor. Berechnet werden AU-Fallzahlen stratifiziert nach Geschlecht und Alter sowie indirekt standardisierte Morbiditätsratios für das Auftreten von AU-Fällen nach Alter und Berufsgruppen stratifiziert.

Ergebnis Beschäftigte in Produktionsberufen mit geringem und mittlerem Qualifikationsniveau sind aufgrund von Varizen besonders häufig arbeitsunfähig. In beiden Geschlechtern treten in allen Altersgruppen mindestens anderthalbmal so viele AU-Fälle wie bei qualifizierten kaufmännischen und Verwaltungsberufen (Referenzgruppe) auf. In der Altersgruppe der 35- bis 44-Jährigen sind Beschäftigte in gering qualifizierten Verwaltungsberufen ebenfalls anderthalbmal häufiger arbeitsunfähig als in der Referenzgruppe. Variationen der Effekte über das Lebensalter sind nachweisbar.

Diskussion/Zusammenfassung Da in Zukunft mehr ältere Erwerbstätige zu erwarten sind (Verschiebung des Rentenalters, bessere medizinische Versorgung, Erhöhung des Anteils der Erwerbstätigkeit bei Älteren), sollte die Prävention im Betrieb (z. B. im Rahmen der arbeitsmedizinischen Vorsorge) verstärkt werden. Ein besonderer Schwerpunkt sollte in Produktionsberufen sowie grundsätzlich in Berufen mit geringer Qualifikation liegen.

\section{ABSTRACT}

Aim/Background Sick leave caused by venous diseases occurs frequently. The occurrence of such sick leave events depends on occupational requirements. The aim is to clarify whether occupation specific differences in the incidence of sick leave events due to the ICD-diagnosis "varicose veins of lower extremities" vary depending on age.

Methods The study is based on secondary analysis of sick leave data from almost all German statutory health insurance providers in 2008. The database consists of sex-stratified aggregated data of 26.2 million compulsorily insured. The number of sick leave events stratified by sex and age were calculated. Indirectly standardized morbidity ratios for the events of sick leave stratified by age and occupational group were estimated. Results Employees in manufacturing occupations with lower and medium skill level are especially often on sick leave because of varicose veins. In both genders, we found in all age groups at least one and a half as many sickness absences cases of 
varicose veins as in qualified sales and administrative occupations (reference group). In the age group of 33-44 year olds, employees in the lower qualified administrative occupations were one and a half time on sick leave as in the reference group. Variations of effects due to age were found.
Discussion/Conclusion As more elderly employees are to be expected in the future (postponement of retirement, improved medical care, increases in the share of older people among gainfully employed persons prevention in the workplace should be strengthened (also in the context of preventive health care). A specific emphasis should be laid on production occupations and low skilled occupations.

\section{Hintergrund und Fragestellung}

\section{Hintergrund}

Herz-Kreislauf-Erkrankungen (HKE) verursachen einen großen Anteil der Morbidität und Mortalität in industrialisierten Ländern (1). In Deutschland bedingen sie ca. 40 \% der Sterbefälle, ca. 15\% der Krankenhausfälle und die höchsten Kosten im Gesundheitswesen $(2,3)$. Volkswirtschaftlich werden dabei häufig nur die Erkrankungen des Herzens und der arteriellen Gefäße betrachtet. Die sozioökonomische Bedeutung der Erkrankungen des Venen- und Lymphsystems ist ebenso beträchtlich. Sie verursachen ca. 5 \% der Krankheitskosten der HKE, fast ebenso viel wie Herzinfarkte (4). Fast ein Drittel ist durch die in der ICD 10 als I83 codierten Varizen der unteren Extremitäten bedingt. Vaskuläre Erkrankungen und insbesondere venöse Erkrankungen sind in ihrer Bedeutung für die Arbeitswelt zu wenig erforscht.

In diesem Beitrag werden basierend auf Arbeitsunfähigkeitsdaten der gesetzlichen Krankenkassen aus dem Jahr 2008 Unterschiede im Auftreten von Arbeitsunfähigkeit bedingt durch Beinvarizen in Abhängigkeit vom Beruf altersunabhängig und stratifiziert für Altersklassen deskriptiv dargestellt.

\section{Bedeutung}

In allgemeinärztlichen Praxen stellen Varizen eine häufige Behandlungsdiagnose (Rang 14) dar (5). Varizen sind eine chronische Erkrankung, die sowohl zu Arbeitsunfähigkeit (AU) als auch zu Frühverrentungen führen können. Sie verursachen ca. 8 \% der AU-Fälle und AU-Tage der HKE (6). Nicht alle Erkrankten haben klinisch relevante Symptome. Unbehandelt führen Varizen häufig zu Komplikationen wie dem chronischen Ödem, trophischen Hautveränderungen, Ulcus cruris, tiefer Veneninsuffizienz, Entzündungen sowie erhöhtem Thromboserisiko.

Operationen an epifaszialen Venen gehören in Deutschland zu den häufigsten Eingriffen. Es wird geschätzt, dass jährlich mehr als 350000 Operationen aufgrund von Varizen durchgeführt werden (7). Im Jahr 2015 gab es insgesamt ca. 93000 vollstationäre Krankenhausfälle (ca. 34000 Männer bzw. 59000 Frauen) aufgrund von Varizen und 140000 vollstationäre Operationen (ca. 49000 Männer bzw. 91000 Frauen). Die Fallzahl nimmt kontinuierlich mit dem Alter zu. Die altersspezifische Fallzahl steigt von 50 Fällen pro 100000 Einwohner bei 15- bis 44-Jährigen auf 162 Fälle pro 100000 Einwohner bei 45- bis 64-Jährigen (8).

\section{Häufigkeiten}

Varizen der unteren Extremitäten sind die häufigste Venenerkrankung. Abhängig von der Definition und dem untersuchten Kollek- tiv werden sehr unterschiedlich hohe Häufigkeiten berichtet. In bevölkerungsbasierten Studien beträgt die Prävalenz zwischen 14\% und 30 \% (9-11). 2015 wurden mehr als 94000 AU-Fälle, 60000 bei Frauen und über 34000 bei Männern, angegeben (6).

Daten aus Deutschland zur Auswirkung auf die Arbeitsfähigkeit liegen bisher nur aus der Tübinger Venenstudie von 1979 vor. Rund 5 \% der an Varizen Erkrankten leiden unter starken Beeinträchtigungen im Beruf. Von ihnen waren $45 \%$ länger als sechs Wochen arbeitsunfähig und $55 \%$ mussten den Arbeitsplatz wechseln, umschulen oder die Arbeit aufgeben (12). Die Rentenversicherung berichtet für 2015 von 58 Berentungen pro 100000 Versicherten aufgrund verminderter Erwerbsfähigkeit bedingt durch Varizen (13).

\section{Risikofaktoren}

Die Ausbildung von Varizen ist ein multifaktorielles Geschehen. Weitgehend anerkannte Risikofaktoren sind Alter, weibliches Geschlecht sowie das Zusammentreffen genetischer Disposition mit mechanischen Faktoren wie langes Stehen, Adipositas oder Schwangerschaften. Weitere allgemeine Einflussfaktoren wie sozialer Status, die Einnahme von oralen Antikontrazeptiva, geringe physische Aktivität, chronische Obstipation, steigende Körpergröße und -gewicht, Arthrose sowie Alkoholkonsum und Rauchen werden teilweise kontrovers diskutiert. Auch Zusammenhänge mit Erkrankungen des arteriellen Gefäßsystems, wie Bluthochdruck und Arteriosklerose werden beschrieben (14-25).

Medizinhistorisch lange bekannt und in epidemiologischen Studien nachgewiesen ist der Zusammenhang zwischen langem Stehen am Arbeitsplatz (über 4 Stunden) und dem Auftreten von Varizen. Auch wenn nicht alle Autoren damit übereinstimmen, wird der Zusammenhang in vielen aktuellen Untersuchungen bestätigt $(10,11,17,22,26,27)$. Vorwiegend sitzende Tätigkeiten werden als mögliches Risiko (17), ohne Einfluss (22) oder protektiv (19) beschrieben. Als weitere berufliche Risikofaktoren werden beruflicher Status als Arbeiter (11), schweres Heben und Tragen (28,29), Arbeiten in geschlossenen Räumen (29), sowie berufliche Exposition gegenüber Hitze und Feuchtigkeit (30) in einzelnen Studien berichtet.

Das Bundesinstitut für Berufsbildung (BIBB) führt regelmäßig in Kooperation mit der Bundesanstalt für Arbeitsschutz und Arbeitsmedizin (BAuA) die BIBB/BAuA-Erwerbstätigenbefragung durch. In der Befragung 2012 (31) geben Beschäftigte (Männer und Frauen), die häufig stehen, doppelt so oft Beschwerden aufgrund geschwollener Beine an wie Beschäftigte, die nie stehen (13,5\% vs. 6,6\%). Schmerzen in den Beinen bzw. Füßen werden fünfmal häufiger (29,2\% vs. 5,9\%) berichtet (eigene Berechnungen). Insgesamt klagen Frauen häufiger über Beschwerden in den Beinen als Männer. 
> Tab. 1 Anzahl der Fälle von Arbeitsunfähigkeit durch „Varizen der unteren Extremitäten“, stratifiziert nach Geschlecht und Alter (10 Jahresklassen), Deutschland 2008.

\begin{tabular}{|c|c|c|c|c|c|c|c|c|c|c|}
\hline \multirow[b]{2}{*}{$\begin{array}{l}\text { Alter } \\
\text { (Jahre) }\end{array}$} & \multicolumn{5}{|l|}{ Männer } & \multicolumn{5}{|l|}{ Frauen } \\
\hline & $\begin{array}{l}\text { Anzahl } \\
\text { Versicherte }\end{array}$ & AU-Fälle & $\begin{array}{l}\text { Fälle/1000 } \\
\text { Versicherte }\end{array}$ & $\begin{array}{l}\text { AU- } \\
\text { Tage }\end{array}$ & Tage/Fall & $\begin{array}{l}\text { Anzahl } \\
\text { Versicherte }\end{array}$ & AU-Fälle & $\begin{array}{l}\text { Fälle/1000 } \\
\text { Versicherte }\end{array}$ & $\begin{array}{l}\text { AU- } \\
\text { Tage }\end{array}$ & Tage/Fall \\
\hline 15 bis 24 & 2155260 & 725 & 0,34 & 8756 & 12,1 & 1777943 & 1091 & 0,61 & 12000 & 11,0 \\
\hline 25 bis 34 & 3149471 & 3040 & 0,97 & 46245 & 15,2 & 2695645 & 5025 & 1,86 & 64908 & 12,9 \\
\hline 35 bis 44 & 3575320 & 7992 & 2,24 & 137167 & 17,2 & 3195389 & 14674 & 4,59 & 219886 & 15,0 \\
\hline 45 bis 54 & 3214339 & 11732 & 3,65 & 214517 & 18,3 & 3237518 & 20873 & 6,45 & 346474 & 16,6 \\
\hline 55 bis 64 & 1635679 & 8798 & 5,38 & 180811 & 20,6 & 1539247 & 13279 & 8,63 & 253799 & 19,1 \\
\hline insgesamt & 13730069 & 32287 & 2,35 & 587496 & 18,2 & 12445742 & 54942 & 4,41 & 897067 & 16,3 \\
\hline
\end{tabular}

\section{Fragestellung}

Bedingt durch die demographische Entwicklung und die verlängerte gesetzliche Lebensarbeitszeit bis zum 67. Lebensjahr ist mit einem Anstieg der AU aufgrund von Beinvarizen zu rechnen, da diese im Alter vermehrt auftreten. Aufgrund unterschiedlicher Beanspruchungen in den Berufen ist es wichtig, den Altersgang der $\mathrm{AU}$ auch berufsabhängig zu kennen.

In den regelmäßig veröffentlichten Statistiken der Krankenkassen und der Bundesregierung über gesetzlich Versicherte werden AUFälle und -Tage für beide Geschlechter aggregiert nach Diagnose-, sowie teilweise auch nach Alters- oder Berufsgruppen berichtet (6). Bisherige Auswertungen der Arbeitsunfähigkeit durch Varizen an den Beinen berücksichtigen weder den Beruf noch Modifikationen im Altersverlauf für das Auftreten von AU durch Varizen.

In diesem Beitrag wird das Auftreten von AU-Fällen aufgrund von Varizen der unteren Extremitäten (183) zwischen verschiedenen Berufsgruppen dargestellt. Alters- und berufsgruppenabhängige Muster werden untersucht. Es wird angenommen, dass in den betroffenen Berufsgruppen das standardisierte Morbiditätsratios (SMR) insbesondere in den jüngeren bis mittleren Altersklassen erhöht ist und eine Annäherung an die Referenzgruppe mit dem Alter erfolgt, da Varizen im jüngeren Alter seltener auftreten und im höheren Alter mit einem Healthy-Worker-Effekt zu rechnen ist.

Die Ergebnisse werden für Männer und Frauen getrennt beschrieben.

\section{Methodik}

\section{Datengrundlage}

Die hier vorgestellte Analyse war Bestandteil eines Forschungsprojekts der BAuA (F2255) mit Sekundärdaten (32). Für dieses Projekt wurden aggregierte Daten von fast allen Kassen der gesetzlichen Krankenversicherung (GKV) zur Arbeitsunfähigkeit in Deutschland für das Kalenderjahr 2008 zusammengestellt. Es lagen Angaben zu insgesamt 26,2 Mio. erwerbstätig Versicherten aus AOK, BKK, IKK, BARMER, TK, DAK, GEK und Knappschaft vor. Die Datenübermittlung erfolgte gruppiert in fünf Altersklassen von 15 bis 64 Jahren.

Die Zahl der AU-Fälle und -Tage zu den 22 häufigsten HKE (Kapitel IX, ICD-10) (33) inklusive der Diagnose I83 Varizen der unte- ren Extremitäten wurden getrennt für beide Geschlechter zur Verfügung gestellt.

Der Datensatz bildet mit insgesamt 13,7 Mio. Männern und 12,5 Mio. Frauen die erwerbstätigen GKV-Pflichtversicherten des Jahres 2008 fast vollständig ab. Am stärksten besetzt ist die Altersklasse der 35- bis 44-Jährigen (3,6 Mio. Männer und 3,2 Mio. Frauen), am niedrigsten die Altersklasse der Erwerbstätigen zwischen 55 und 64 Jahren (1,6 Mio. Männer, 1,5 Mio. Frauen) (॰ Tab. 1).

\section{Datenauswertung}

Angaben zum Beruf waren entsprechend der Klassifikation der Berufe (34) für die dreistellige Berufsordnung kodiert. Die Berufsgruppen wurden entsprechend der Klassifikation von Blossfeld (35) gebildet. Diese fasst die 336 Berufe sekundär in 12 Berufsgruppen entsprechend Qualifikation und Tätigkeitsanforderungen zusammen. Eine Auflistung mit Angabe zur Gruppengröße für Männer und Frauen findet sich in $>$ Tab. 2 und $>$ Tab. 3 . Zur genauen Beschreibung siehe Liebers et al. 2016 (32).

In diesem Beitrag werden nur die Ergebnisse für AU-Fälle vorgestellt. Für die Aussagen zu relativen Häufigkeiten wurden die Anzahl der AU-Fälle je Diagnose auf die Anzahl der Versicherten pro Altersklasse und Beruf (bzw. Berufsgruppe) bezogen. Als Effektschätzer wurden SMR als Verhältnis der beobachteten zur erwarteten Anzahl berechnet. Das SMR wird als relatives Risiko interpretiert.

- Das generelle berufsgruppenspezifische SMR ist indirekt standardisiert. Berücksichtigt wurden Alter und Kassenzugehörigkeit als Einflussfaktoren. Alle Berechnungen erfolgten geschlechtsstratifiziert. Referenzgruppe für die Auswertung waren die Bürofachkräfte bzw. die qualifizierten kaufmännischen und Verwaltungsberufe. Die Auswahl erfolgte aufgrund der hohen Anzahl an Beschäftigten in beiden Geschlechtern und der teilweise geringen physischen Belastung.

- Für die Berechnung der berufsgruppenspezifischen SMR im Altersverlauf erfolgte die Berechnung jeweils für Probanden einer Berufsgruppe innerhalb einer der fünf Altersklassen im Vergleich zu altersgleichen Probanden der Referenzberufsgruppe. Die indirekte Standardisierung berücksichtigt in diesem Fall nur die Kassenzugehörigkeit.

Für die SMR wurden exakte Konfidenzintervalle (KI) berechnet. Aufgrund des multiplen Testens wurden erweiterte 99,99\% KI verwen- 
- Tab. 2 Standardisiertes Morbiditätsratio mit 99,99\% Konfidenzintervall (KI) der alters- und berufsgruppenspezifischen Arbeitsunfähigkeit aufgrund der Diagnose „Varizen der unteren Extremitäten“ von pflichtversicherten Erwerbstätigen in Deutschland 2008, Männer. Standardisiert für die gesetzlichen Krankenkassen.

\begin{tabular}{|c|c|c|c|c|c|c|c|}
\hline \multirow[b]{2}{*}{ Berufsgruppe nach Blossfeld $1985^{*}$} & \multirow[t]{2}{*}{ Versicherte } & \multicolumn{6}{|c|}{ Standardisiertes Morbiditätsratio [99,99\% KI] } \\
\hline & & 15-24 Jahre & 25-34 Jahre & 35-44 Jahre & 45-54 Jahre & 55-64 Jahre & gesamt \\
\hline qualifizierte manuelle Berufe & 3538972 & $\begin{array}{l}1,21 \\
{[0,96-1,49]}\end{array}$ & $\begin{array}{l}1,94 \\
{[1,71-2,20]}\end{array}$ & $\begin{array}{l}1,74 \\
{[1,61-1,88]}\end{array}$ & $\begin{array}{l}1,60 \\
{[1,50-1,71]}\end{array}$ & $\begin{array}{l}1,50 \\
{[1,39-1,61]}\end{array}$ & $\begin{array}{l}1,61 \\
{[1,55-1,68]}\end{array}$ \\
\hline gering qualifizierte manuelle Berufe & 2951981 & $\begin{array}{l}1,19 \\
{[0,84-1,61]}\end{array}$ & $\begin{array}{l}1,97 \\
{[1,72-2,25]}\end{array}$ & $\begin{array}{l}1,77 \\
{[1,63-1,92]}\end{array}$ & $\begin{array}{l}1,52 \\
{[1,42-1,62]}\end{array}$ & $\begin{array}{l}1,55 \\
{[1,43-1,68]}\end{array}$ & $\begin{array}{l}1,61 \\
{[1,55-1,68]}\end{array}$ \\
\hline gering qualifizierte Verwaltungsberufe & 542660 & $\begin{array}{l}1,08 \\
{[0,48-2,04]}\end{array}$ & $\begin{array}{l}1,20 \\
{[0,82-1,70]}\end{array}$ & $\begin{array}{l}1,47 \\
{[1,18-1,82]}\end{array}$ & $\begin{array}{l}1,20 \\
{[0,97-1,47]}\end{array}$ & $\begin{array}{l}1,11 \\
{[0,86-1,41]}\end{array}$ & $\begin{array}{l}1,24 \\
{[1,10-1,40]}\end{array}$ \\
\hline $\begin{array}{l}\text { gering qualifizierte Dienstleistungs- } \\
\text { berufe }\end{array}$ & 2333934 & $\begin{array}{l}1,29 \\
{[0,80-1,96]}\end{array}$ & $\begin{array}{l}1,70 \\
{[1,43-2,01]}\end{array}$ & $\begin{array}{l}1,18 \\
{[1,06-1,31]}\end{array}$ & $\begin{array}{l}1,15 \\
{[1,06-1,25]}\end{array}$ & $\begin{array}{l}1,11 \\
{[1,01-1,21]}\end{array}$ & $\begin{array}{l}1,18 \\
{[1,12-1,24]}\end{array}$ \\
\hline qualifizierte Dienstleistungsberufe & 318130 & $\begin{array}{l}0,58 \\
{[0,08-1,97]}\end{array}$ & $\begin{array}{l}1,25 \\
{[0,74-1,96]}\end{array}$ & $\begin{array}{l}1,12 \\
{[0,81-1,51]}\end{array}$ & $\begin{array}{l}1,17 \\
{[0,90-1,49]}\end{array}$ & $\begin{array}{l}1,19 \\
{[0,87-1,59]}\end{array}$ & $\begin{array}{l}1,16 \\
{[0,99-1,34]}\end{array}$ \\
\hline Agrarberufe & 309992 & $\begin{array}{l}1,12 \\
{[0,40-2,41]}\end{array}$ & $\begin{array}{l}1,47 \\
{[0,83-2,38]}\end{array}$ & $\begin{array}{l}1,34 \\
{[0,98-1,77]}\end{array}$ & $\begin{array}{l}1,06 \\
{[0,81-1,35]}\end{array}$ & $\begin{array}{l}1,03 \\
{[0,75-1,38]}\end{array}$ & $\begin{array}{l}1,15 \\
{[0,98-1,33]}\end{array}$ \\
\hline Techniker & 667643 & $\begin{array}{l}0,35 \\
{[0,03-1,27]}\end{array}$ & $\begin{array}{l}1,08 \\
{[0,70-1,57]}\end{array}$ & $\begin{array}{l}1,39 \\
{[1,14-1,68]}\end{array}$ & $\begin{array}{l}1,05 \\
{[0,88-1,25]}\end{array}$ & $\begin{array}{l}0,97 \\
{[0,79-1,17]}\end{array}$ & $\begin{array}{l}1,09 \\
{[0,99-1,21]}\end{array}$ \\
\hline Semiprofessionen & 404730 & $\begin{array}{l}1,11 \\
{[0,34-2,60]}\end{array}$ & $\begin{array}{l}1,40 \\
{[0,89-2,10]}\end{array}$ & $\begin{array}{l}1,24 \\
{[0,94-1,60]}\end{array}$ & $\begin{array}{l}0,93 \\
{[0,72-1,17]}\end{array}$ & $\begin{array}{l}1,07 \\
{[0,81-1,38]}\end{array}$ & $\begin{array}{l}1,08 \\
{[0,94-1,24]}\end{array}$ \\
\hline Manager & 221965 & $\begin{array}{l}1,27 \\
{[0,05-5,83]}\end{array}$ & $\begin{array}{l}0,76 \\
{[0,34-1,42]}\end{array}$ & $\begin{array}{l}0,56 \\
{[0,31-0,92]}\end{array}$ & $\begin{array}{l}0,90 \\
{[0,62-1,27]}\end{array}$ & $\begin{array}{l}0,93 \\
{[0,65-1,29]}\end{array}$ & $\begin{array}{l}0,82 \\
{[0,66-1,01]}\end{array}$ \\
\hline Ingenieure & 331193 & $\begin{array}{l}0,79 \\
{[0,01-4,35]}\end{array}$ & $\begin{array}{l}0,60 \\
{[0,32-1,01]}\end{array}$ & $\begin{array}{l}0,70 \\
{[0,46-1,01]}\end{array}$ & $\begin{array}{l}0,83 \\
{[0,57-1,15]}\end{array}$ & $\begin{array}{l}0,82 \\
{[0,56-1,15]}\end{array}$ & $\begin{array}{l}0,76 \\
{[0,62-0,91]}\end{array}$ \\
\hline Professionen & 133762 & $\begin{array}{l}0,00 \\
{[0,00-0,00]}\end{array}$ & $\begin{array}{l}0,29 \\
{[0,07-0,76]}\end{array}$ & $\begin{array}{l}0,50 \\
{[0,20-1,00]}\end{array}$ & $\begin{array}{l}0,65 \\
{[0,30-1,22]}\end{array}$ & $\begin{array}{l}1,05 \\
{[0,53-1,84]}\end{array}$ & $\begin{array}{l}0,61 \\
{[0,41-0,86]}\end{array}$ \\
\hline nicht zuordenbar & 345331 & $\begin{array}{l}0,74 \\
{[0,37-1,29]}\end{array}$ & $\begin{array}{l}1,03 \\
{[0,53-1,80]}\end{array}$ & $\begin{array}{l}0,81 \\
{[0,46-1,31]}\end{array}$ & $\begin{array}{l}0,74 \\
{[0,47-1,09]}\end{array}$ & $\begin{array}{l}0,42 \\
{[0,21-0,74]}\end{array}$ & $\begin{array}{l}0,70 \\
{[0,55-0,88]}\end{array}$ \\
\hline qualifizierte Verwaltungsberufe & 1636398 & $\begin{array}{l}1 \\
\text { (Referenz) }\end{array}$ & $\begin{array}{l}1 \\
\text { (Referenz) }\end{array}$ & $\begin{array}{l}1 \\
\text { (Referenz) }\end{array}$ & $\begin{array}{l}1 \\
\text { (Referenz) }\end{array}$ & $\begin{array}{l}1 \\
\text { (Referenz) }\end{array}$ & $\begin{array}{l}1 \\
\text { (Referenz) }\end{array}$ \\
\hline AU-Fälle/10 000 in Referenz (roh) & & 3 & 6 & 14 & 26 & 37 & 15 \\
\hline
\end{tabular}

det. Als signifikant werden Effektschätzer bewertet, deren KI des SMR die 1 nicht einschließt.

Bei der Berechnung konnten nur Angaben zu Geschlecht, Altersklasse und Krankenkasse als Kovariablen berücksichtigt werden. Weitere Angaben wie z. B. zu sozioökonomischem Status, Einkommen, Bildung, Konstitution oder Disposition lagen nicht vor.

Für die Verwaltung und Auswertung, wurde die relationale Datenbank Microsoft Access 2003 verwendet.

\section{Ergebnisse}

\section{Altersspezifische AU-Ereignissen}

Im Jahr 2008 wurden 87229 Fälle von AU aufgrund von Varizen an den Beinen bei Beschäftigten im Alter von 15 bis 64 Jahren beobachtet. Bei den Männern führten 32287 AU-Fälle (2,1/1000 Beschäftigte) zu 587496 AU-Tagen (42,8/1000 Beschäftigte). Bei den Frauen führten die 54942 AU-Fälle (4,1/1000 Beschäftigte) zu 897067 AU-Tagen (72,1/1000 Beschäftigte). Die durchschnittliche Dauer einer AU betrug bei Männern 18,2 und bei Frauen 16,3 Tage. Sowohl Männer und als auch Frauen zeigten einen altersabhängigen Anstieg der AU. Ebenso nahm in beiden Geschlechtern die absolute Anzahl der AU-Fälle mit dem Alter zu ( $\triangleright$ Tab. 1). Genauere Ausführungen finden sich bei Liebers 2016 (32).

\section{Berufsgruppenspezifische Arbeitsunfähigkeit}

Bei Beschäftigten in qualifizierten manuellen Berufen ( $\sigma^{\star}$ SMR 1,61; KI: $[1,55-1,68]$ und o SMR $1,73[1,61-1,84])$, in gering qualifizierten manuellen Berufen ( $\sigma^{*}$ SMR 1,61 [1,55-1,68] und o SMR 1,51 $[1,43-1,58])$, in gering qualifizierten Verwaltungsberufen ( ${ }^{*}$ SMR $1,24[1,10-1,40]$ und o SMR $1,39[1,34-1,45])$ sowie in gering qualifizierten Dienstleistungsberufen ( $\sigma^{*}$ SMR 1,18 [1,12-1,24] und + SMR 1,35 [1,30-1,41]) traten mehr AU-Fälle aufgrund von Varizen als in der Referenzgruppe auf. Anders als bei den Männern tra- 
- Tab. 3 Standardisiertes Morbiditätsratio mit 99,99\% Konfidenzintervall (KI) der alters- und berufsgruppenspezifischen Arbeitsunfähigkeit aufgrund der Diagnose „Varizen der unteren Extremitäten“ von pflichtversicherten Erwerbstätigen in Deutschland 2008, Frauen. Standardisiert für die gesetzlichen Krankenkassen.

\begin{tabular}{|c|c|c|c|c|c|c|c|}
\hline \multirow[b]{2}{*}{ Berufsgruppe nach Blossfeld 1985* } & \multirow[t]{2}{*}{ Versicherte } & \multicolumn{6}{|c|}{ Standardisiertes Morbiditätsratio [99,99\% KI] } \\
\hline & & 15-24 Jahre & 25-34 Jahre & 35-44 Jahre & 45-54 Jahre & 55-64 Jahre & gesamt \\
\hline qualifizierte manuelle Berufe & 482647 & $\begin{array}{l}1,29 \\
{[0,77-2,01]}\end{array}$ & $\begin{array}{l}2,16 \\
{[1,70-2,70]}\end{array}$ & $\begin{array}{l}1,72 \\
{[1,49-1,96]}\end{array}$ & $\begin{array}{l}1,68 \\
{[1,50-1,87]}\end{array}$ & $\begin{array}{l}1,74 \\
{[1,53-1,97]}\end{array}$ & $\begin{array}{l}1,73 \\
{[1,61-1,84]}\end{array}$ \\
\hline gering qualifizierte manuelle Berufe & 842681 & $\begin{array}{l}1,04 \\
{[0,59-1,66]}\end{array}$ & $\begin{array}{l}1,91 \\
{[1,56-2,31]}\end{array}$ & $\begin{array}{l}1,52 \\
{[1,37-1,68]}\end{array}$ & $\begin{array}{l}1,44 \\
{[1,33-1,56]}\end{array}$ & $\begin{array}{l}1,54 \\
{[1,40-1,70]}\end{array}$ & $\begin{array}{l}1,51 \\
{[1,43-1,58]}\end{array}$ \\
\hline gering qualifizierte Verwaltungsberufe & 1719310 & $\begin{array}{l}1,25 \\
{[0,92-1,66]}\end{array}$ & $\begin{array}{l}1,62 \\
{[1,40-1,85]}\end{array}$ & $\begin{array}{l}1,47 \\
{[1,36-1,58]}\end{array}$ & $\begin{array}{l}1,31 \\
{[1,22-1,40]}\end{array}$ & $\begin{array}{l}1,38 \\
{[1,27-1,50]}\end{array}$ & $\begin{array}{l}1,39 \\
{[1,34-1,45]}\end{array}$ \\
\hline $\begin{array}{l}\text { gering qualifizierte Dienstleistungs- } \\
\text { berufe }\end{array}$ & 1448912 & $\begin{array}{l}1,21 \\
{[0,83-1,69]}\end{array}$ & $\begin{array}{l}1,60 \\
{[1,35-1,87]}\end{array}$ & $\begin{array}{l}1,34 \\
{[1,23-1,46]}\end{array}$ & $\begin{array}{l}1,30 \\
{[1,22-1,39]}\end{array}$ & $\begin{array}{l}1,39 \\
{[1,29-1,50]}\end{array}$ & $\begin{array}{l}1,35 \\
{[1,30-1,41]}\end{array}$ \\
\hline qualifizierte Dienstleistungsberufe & 1282075 & $\begin{array}{l}1,30 \\
{[0,99-1,67]}\end{array}$ & $\begin{array}{l}1,27 \\
{[1,08-1,48]}\end{array}$ & $\begin{array}{l}1,23 \\
{[1,10-1,37]}\end{array}$ & $\begin{array}{l}1,20 \\
{[1,09-1,32]}\end{array}$ & $\begin{array}{l}1,25 \\
{[1,10-1,42]}\end{array}$ & $\begin{array}{l}1,23 \\
{[1,16-1,30]}\end{array}$ \\
\hline Agrarberufe & 130253 & $\begin{array}{l}0,60 \\
{[0,12-1,71]}\end{array}$ & $\begin{array}{l}1,34 \\
{[0,76-2,18]}\end{array}$ & $\begin{array}{l}1,19 \\
{[0,86-1,59]}\end{array}$ & $\begin{array}{l}1,19 \\
{[0,89-1,55]}\end{array}$ & $\begin{array}{l}1,24 \\
{[0,84-1,77]}\end{array}$ & $\begin{array}{l}1,19 \\
[1,0-1,40]]\end{array}$ \\
\hline Techniker & 358308 & $\begin{array}{l}0,84 \\
{[0,29-1,84]}\end{array}$ & $\begin{array}{l}1,48 \\
{[1,08-1,96]}\end{array}$ & $\begin{array}{l}1,13 \\
{[0,93-1,36]}\end{array}$ & $\begin{array}{l}1,08 \\
{[0,91-1,28]}\end{array}$ & $\begin{array}{l}1,16 \\
{[0,92-1,45]}\end{array}$ & $\begin{array}{l}1,15 \\
{[1,03-1,27]}\end{array}$ \\
\hline Semiprofessionen & 1851860 & $\begin{array}{l}0,99 \\
{[0,69-1,37]}\end{array}$ & $\begin{array}{l}1,34 \\
{[1,16-1,53]}\end{array}$ & $\begin{array}{l}1,27 \\
{[1,17-1,38]}\end{array}$ & $\begin{array}{l}1,20 \\
{[1,12-1,28]}\end{array}$ & $\begin{array}{l}1,24 \\
{[1,13-1,36]}\end{array}$ & $\begin{array}{l}1,23 \\
{[1,18-1,29]}\end{array}$ \\
\hline Manager & 245286 & $\begin{array}{l}0,61 \\
{[0,10-1,94]}\end{array}$ & $\begin{array}{l}0,68 \\
{[0,43-1,03]}\end{array}$ & $\begin{array}{l}0,70 \\
{[0,51-0,93]}\end{array}$ & $\begin{array}{l}0,83 \\
{[0,63-1,06]}\end{array}$ & $\begin{array}{l}1,00 \\
{[0,72-1,35]}\end{array}$ & $\begin{array}{l}0,80 \\
{[0,68-0,93]}\end{array}$ \\
\hline Ingenieure & 107437 & $\begin{array}{l}0,45 \\
{[0,00-5,59]}\end{array}$ & $\begin{array}{l}0,94 \\
{[0,54-1,52]}\end{array}$ & $\begin{array}{l}0,61 \\
{[0,37-0,94]}\end{array}$ & $\begin{array}{l}0,84 \\
{[0,52-1,27]}\end{array}$ & $\begin{array}{l}0,78 \\
{[0,34-1,50]}\end{array}$ & $\begin{array}{l}0,77 \\
{[0,59-0,97]}\end{array}$ \\
\hline Professionen & 223620 & $\begin{array}{l}0,57 \\
{[0,00-4,16]}\end{array}$ & $\begin{array}{l}0,60 \\
{[0,38-0,91]}\end{array}$ & $\begin{array}{l}0,74 \\
{[0,54-0,98]}\end{array}$ & $\begin{array}{l}0,81 \\
{[0,59-1,07]}\end{array}$ & $\begin{array}{l}0,88 \\
{[0,57-1,29]}\end{array}$ & $\begin{array}{l}0,75 \\
{[0,63-0,89]}\end{array}$ \\
\hline nicht zuordenbar & 249508 & $\begin{array}{l}0,71 \\
{[0,38-1,20]}\end{array}$ & $\begin{array}{l}0,70 \\
{[0,38-1,18]}\end{array}$ & $\begin{array}{l}0,53 \\
{[0,32-0,81]}\end{array}$ & $\begin{array}{l}0,61 \\
{[0,40-0,89]}\end{array}$ & $\begin{array}{l}0,60 \\
{[0,32-1,03]}\end{array}$ & $\begin{array}{l}0,62 \\
{[0,49-0,76]}\end{array}$ \\
\hline qualifizierte Verwaltungsberufe & 3509418 & $\begin{array}{l}1 \\
\text { (Referenz) }\end{array}$ & $\begin{array}{l}1 \\
\text { (Referenz) }\end{array}$ & $\begin{array}{l}1 \\
\text { (Referenz) }\end{array}$ & $\begin{array}{l}1 \\
\text { (Referenz) }\end{array}$ & $\begin{array}{l}1 \\
\text { (Referenz) }\end{array}$ & $\begin{array}{l}1 \\
\text { (Referenz) }\end{array}$ \\
\hline $\begin{array}{l}\text { AU-Fälle/10 } 000 \text { in Referenzgruppe } \\
\text { (roh) }\end{array}$ & & 5 & 14 & 37 & 52 & 63 & 34 \\
\hline
\end{tabular}

*Bezeichnung der Berufsgruppen von Blossfeld leicht modifiziert: Ersetzungen: „einfach“ durch „gering qualifiziert“, „Dienste“ durch „Dienstleistungsberufe“, „kaufmännische und Verwaltungsberufe“ durch „Verwaltungsberufe“

ten bei Frauen auch in den Berufsgruppen der Semiprofessionen ${ }^{1}$ (SMR 1,23 [1,18-1,29]), der qualifizierten Dienstleistungsberufe (SMR 1,23 [1,16-1,30]), der Agrarberufe (SMR 1,19 [1,00-1,40]) sowie der Techniker (SMR 1,15 [1,03-1,27]) mehr AU-Fälle als in der Referenzgruppe auf ( $\bullet$ Tab. 2 und $\triangleright$ Tab. 3). Die folgenden altersabhängigen Untersuchungen wurden für die hier genannten Berufsgruppen durchgeführt.

Für beide Geschlechter konnte bestätigt werden, dass einzelne Berufe aus den Berufsgruppen der manuellen Berufe und der gering qualifizierten Dienstleistungs- und Verwaltungsberufe ein erhöhtes Risiko aufweisen. Mit 2- bzw. 3-mal mehr AU-Fällen pro 1000

1 Semiprofessionen: Dienstleistungsberufe, die sich durch eine Verwissenschaftlichung der Berufspositionen auszeichnen, z. B. Krankenschwestern, Sozialarbeiter, Sozialpädagogen, Real- und Volksschullehrer im Gegensatz zu Professionen: Freie Berufe und hochqualifizierte Dienstleistungsberufe, z. B. Ärzte, Zahnärzte, Apotheker, Richter, Gymnasiallehrer, Sozial- und Geisteswissenschaftler
Versicherte als bei Bürofachkräften waren sowohl Männer als auch Frauen in folgenden Einzelberufen besonders betroffen: Konditoren (SMR ox 3,08 bzw. 92,74 ), Backwarenhersteller (SMR ox 2,77; ㅇ 2,21) sowie Kunststoffverarbeiter (SMR ox 1,94; ㅇ 1,95). Ergebnisse nicht dargestellt, ausführlich siehe Liebers et al. 2016 (32).

\section{Alters- und berufsgruppenspezifische Arbeitsunfähigkeit}

Die Häufigkeit des Auftretens von AU-Fällen aufgrund der Diagnose 183 stieg in allen Berufsgruppen mit den Altersklassen an.

In beiden Geschlechtern wurden bei Betrachtung der einzelnen Altersklassen bei den 15- bis 24-Jährigen in allen Berufsgruppen sehr wenige AU-Fälle beobachtet. Keine Berufsgruppe wies signifikant mehr AU-Fälle als die Referenzgruppe auf.

In den drei Altersklassen von 25 bis 54 Jahren zeigten sich bei den Männern durchgängig signifikant vermehrt AU-Fälle in qualifizierten sowie gering qualifizierten manuellen Berufen und in ge- 


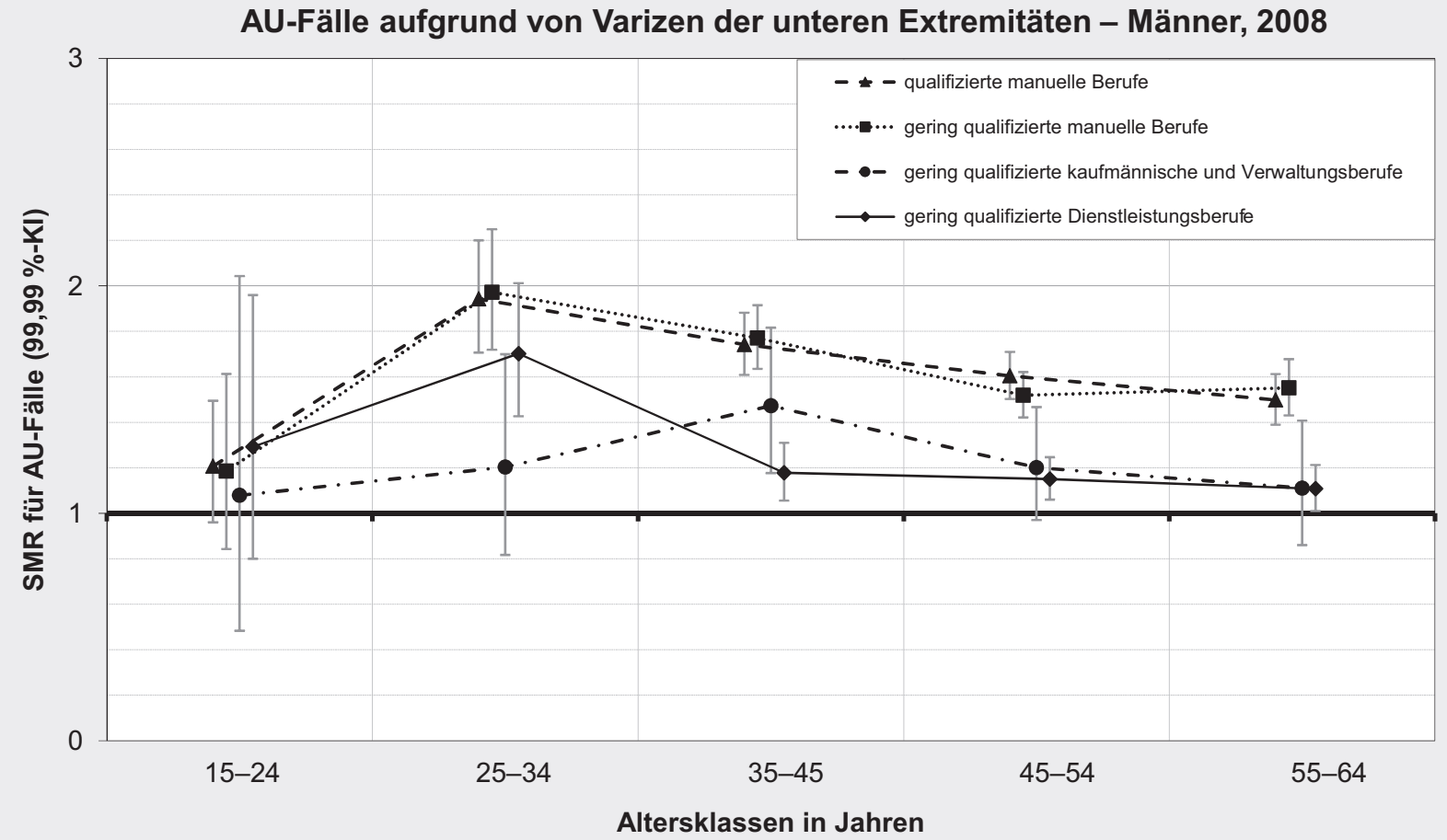

-Abb. 1 Standardmorbiditätsratio (SMR) für das Auftreten von Arbeitsunfähigkeitsfällen (AU-Fälle) im Altersverlauf aufgrund von Varizen der unteren Extremitäten bei Männern für Berufsgruppen mit altersunabhängig erhöhter AU-Fallzahl im Vergleich zu gleichaltrigen Beschäftigten in qualifizierten kaufmännischen und Verwaltungsberufen (Referenz).

ring qualifizierten Dienstleistungsberufen, entsprechend den Ergebnissen der altersunabhängigen Untersuchung. Auffällig waren die hohen Werte in der Altersklasse der 25- bis 34-Jährigen in diesen Berufsgruppen im Vergleich zur Referenzgruppe. In der höchsten Altersklasse ( 55 bis 64 Jahre) traten bei manuellen Berufen weiterhin wesentlich mehr AU-Fälle als in der Referenzgruppe auf. In den anderen Berufsgruppen glich sich das Risiko dem der qualifizierten Verwaltungsberufe (Referenz) an. Bei den gering qualifizierten kaufmännischen und Verwaltungsberufen bestanden nur in der mittleren Altersklasse ( 35 bis 44 Jahre) signifikant mehr AUFälle als in der Referenzgruppe (siehe $>$ Abb. 1).

Bei den Frauen zeigten die Altersklassen ab 25 Jahren signifikant mehr AU für qualifizierte sowie gering qualifizierte manuelle Berufe, qualifizierte sowie gering qualifizierte Dienstleistungsberu$\mathrm{fe}$, Semiprofessionen, gering qualifizierte Verwaltungsberufe und Techniker als die Referenzgruppe analog den altersunabhängigen Ergebnissen (siehe $\mathbf{A}$ Abb. 2).

Die meisten AU-Fälle in der höchsten Altersklasse traten wie bei den Männern in den manuellen Berufen und zusätzlich in den gering qualifizierten Dienstleistungs- sowie Verwaltungsberufen auf. Auffällig waren (wie bei den Männern auch) relativ viele AU-Fälle in der Altersklasse der 25- bis 34-Jährigen in den manuellen Berufen, den gering qualifizierten Dienstleistungsberufen und zusätzlich in den gering qualifizierten Verwaltungsberufen im Vergleich zur Berufsgruppe der qualifizierten kaufmännischen und Verwaltungsberufe. Auch bei Beschäftigten in Semiprofessionen, qualifizierten Dienstleistungsberufen sowie als Techniker traten in die- ser Altersgruppe mehr AU-Fälle als in der Referenzgruppe auf. Das Risiko für AU-Fälle durch Varizen blieb trotz relativen Rückgangs auch mit steigendem Alter gegenüber der Referenz erhöht. In den Agrarberufen bestand in keiner Altersklasse ein signifikant unterschiedliches Ergebnis zu der Referenzgruppe (nicht abgebildet).

Eine differenziertere Auswertung der fünf Altersklassen bezogen auf Einzelberufe war aufgrund der geringen Zellenbesetzung nicht möglich.

\section{Diskussion}

\section{Ergebniszusammenfassung}

Die berufsbezogene Auswertung von AU-Daten informiert vorrangig über das Ausmaß der sozialen Betroffenheit der Beschäftigten in verschiedenen Berufen und Berufsgruppe. Viele Berufe sind trotz Varizen gut ausführbar, während in anderen Berufen, abhängig von der Arbeitsaufgabe, eine Arbeitsausübung nur bedingt möglich ist. Können Beschäftigte ihre Tätigkeit aufgrund der Beschwerden nicht mehr oder nur unter weiterer Verschlechterung ausüben, kommt es zu AU. Varizen können, wie andere HKE auch, zu langer AU und sogar zur Aufgabe der beruflichen Tätigkeit führen (12).

Die Analyse zum berufs- und altersabhängigen Auftreten von AU aufgrund von Beinvarizen wurde mit aggregierten Sekundärdaten durchgeführt. Da ca. $90 \%$ der gesetzlich pflichtversicherten Erwerbstätigen erfasst wurden, sind die Daten als repräsentativ anzusehen. Die ausgewählte Diagnose zeigte erwartungsge- 
AU-Fälle aufgrund von Varizen der unteren Extremitäten - Frauen, 2008

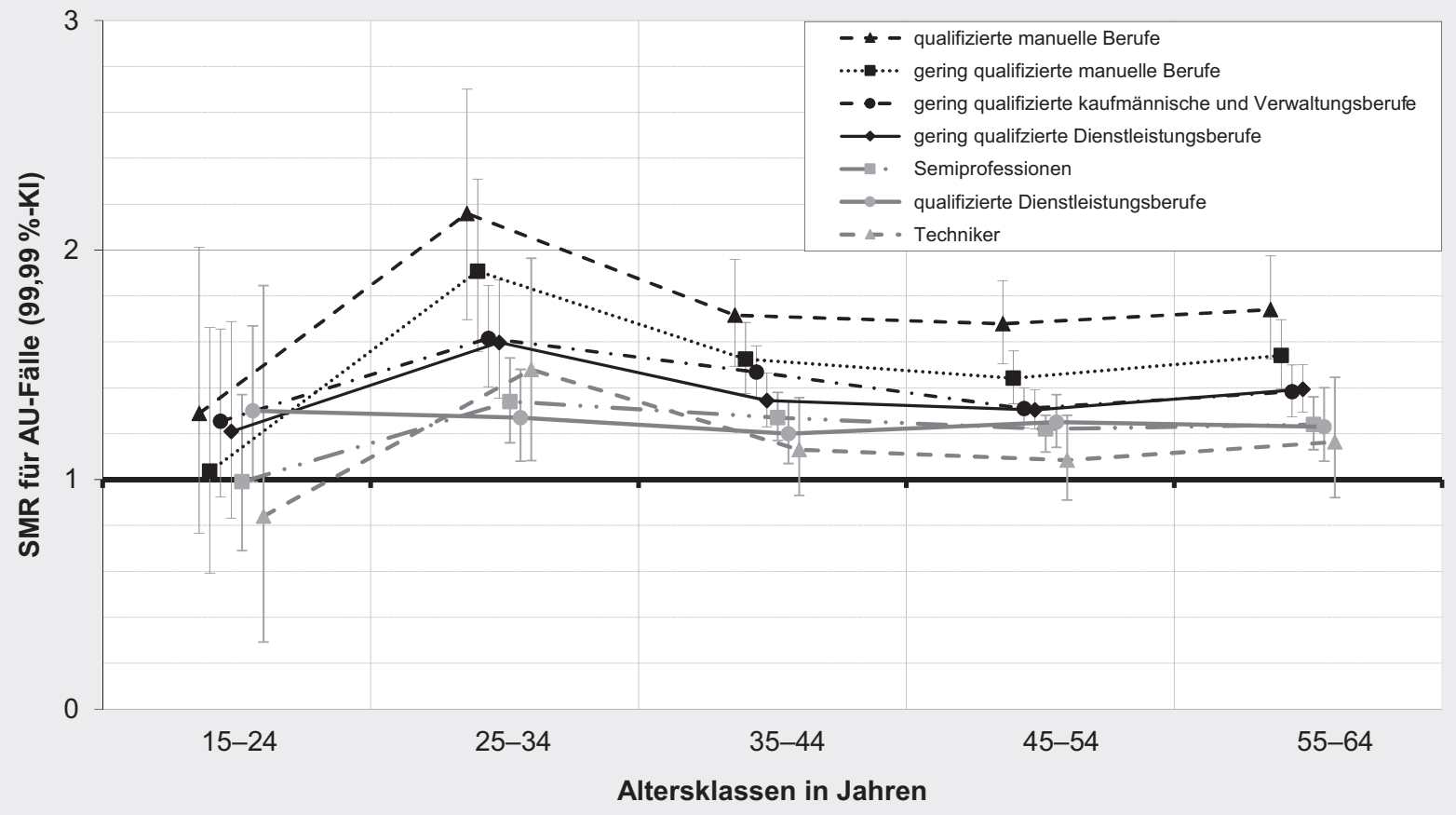

- Abb. 2 Standardmorbiditätsratio (SMR) für das Auftreten von Arbeitsunfähigkeitsfällen (AU-Fälle) im Altersverlauf aufgrund von Varizen der unteren Extremitäten bei Frauen für Berufsgruppen mit altersunabhängig erhöhter AU-Fallzahl im Vergleich zu gleichaltrigen Beschäftigten in qualifizierten kaufmännischen und Verwaltungsberufen (Referenz).

mäß einen starken absoluten und relativen Anstieg von AU-Fällen mit dem Alter. Dies entspricht den bereits vorliegenden Ergebnissen zu anderen HKE (36). Obwohl bei Jugendlichen und jungen Erwachsenen nur geringe Unterschiede in der Häufigkeit von Varizen zwischen den Geschlechtern bekannt sind (37), traten bei Frauen bereits ab der jüngsten Altersklasse mehr AU-Fälle als bei Männern auf. Die Berufsgruppen qualifizierte und gering qualifizierte manuelle Berufe, gering qualifizierte Dienstleistungsberufe sowie gering qualifizierte kaufmännische und Verwaltungsberufe zeigten für beide Geschlechter altersunabhängig erhöhte Risiken gegenüber der Referenz für das Auftreten von AU-Fällen. Bei den Frauen besteht diese Erhöhung auch bei Semiprofessionen, qualifizierten Dienstleistungsberufen, Agrarberufen und bei Technikern.

Varizen sind eine chronische, sich progredient entwickelnde Erkrankungen, die und deren Folgen bei Jüngeren seltener auftreten. Durch die kurze Zeit im Beruf kann von einer geringen berufsbedingten Risikoerhöhung der Erkrankung und der AU ausgegangen werden. Die relative Häufigkeit von Varizen steigt mit dem Alter an. Die Summierung der Belastungen über die Arbeitsjahre kann zu einer weiteren Erhöhung der relativen Häufigkeit von AU-Fällen bei Erwerbstätigen beitragen. Die Stratifizierung der Auswertung nach Altersklassen bietet eine differenzierte Darstellung der Betroffenheit durch AU.

In der jüngsten Altersklasse bestanden für fast alle Berufsgruppen unpräzise Schätzwerte aufgrund von geringen Fallzahlen. In fast allen Berufsgruppen war ein deutlicher Anstieg der AU-Fälle verglichen mit der Gruppe der qualifizierten kaufmännischen und
Verwaltungsberufe von der Altersklasse der 15- bis 24-Jährigen zu den 25- bis 34-Jährigen zu beobachten.

Dies wurde bei Männern bereits in Untersuchungen zu anderen Erkrankungen (Myokardinfarkt, Rückenschmerzen und Gonarthrose) gesehen. In den genannten Diagnosen konnte bei den Frauen kein derartiger Anstieg gefunden werden. Bei der arteriellen Hypertonie gilt dies für beide Geschlechter $(36,38)$.

Das Risiko aufgrund von Varizen arbeitsunfähig zu werden war ab dem Alter von 35 Jahren relativ konstant. Bestehende Unterschiede zwischen den Berufsgruppen blieben bestehen. Beschäftigte in manuellen Berufen wiesen aber auch in der höchsten Altersklasse die höchste AU auf.

\section{Fehlerdiskussion}

Die Ergebnisse der altersbezogenen Auswertung beruhen auf Querschnittsdaten und stellen keine Verlaufsbeobachtung einer Kohorte dar (39). Eine kausale Interpretation zwischen beruflicher Exposition und Erkrankung ist nicht möglich. Außer Alter, Geschlecht und Kassenzugehörigkeit konnten keine weiteren Einflussfaktoren berücksichtigt werden. Als wichtige Störgrößen sind sozio-ökonomischer Status und Lebensstilfaktoren anzusehen.

Der Auswertung lagen aggregierte, keine personenbezogenen AU-Daten zugrunde. Aus der Anzahl von AU-Fällen kann nicht auf die Zahl der betroffenen Versicherten geschlossen werden, da mehrere AU-Fälle pro Versichertem pro Jahr auftreten können. Zur weitergehenden Interpretation der Studienergebnisse wird auf die Fehlerdiskussion in der vorliegenden Literatur (32) bzgl. des Be- 
rufs als Surrogat für arbeitsbezogene und außerberufliche Risiken und die Möglichkeiten der Fehlklassifikation bei der Berufskodierung verwiesen.

Der starke Risikoanstieg bei den 25- bis 34-Jährigen beruht u. a. auf relativ wenigen AU-Fällen in der Vergleichsgruppe in dieser Altersklasse. Es besteht die Möglichkeit, dass das Auftreten von Varizen in diesem Alter gleichmäßig zwischen den Berufsgruppen verteilt ist, jedoch nur in beanspruchten Berufsgruppen Erwerbstätige infolge dieser Diagnose arbeitsunfähig werden. Ebenso ist es möglich, dass in den betroffenen Berufsgruppen die Varizen früher auftreten und deshalb in dieser Altersgruppe bereits zu AU führt. In den höheren Altersklassen kann man davon ausgehen, dass z. B. durch langjährige stehende Tätigkeiten mehr Varizen und Komplikationen verursacht werden (27).

Healthy-Worker-Effekte sind ebenfalls zu bedenken. Beschäftigte, die schon in ihrer Jugend an Varizen leiden, werden seltener Berufe mit zusätzlichen Risikofaktoren - insbesondere Stehen - aufnehmen oder in diesen verbleiben.

Aus den vorliegenden Daten kann nicht auf die Ursache (ambulante oder stationäre bzw. operative oder konservative Therapie) der AU geschlossen werden. Bei über 75000 stationären Operationen in der Altersgruppe der 15 bis 64-Jährigen (8) und wahrscheinlich ebenso vieler ambulanter Operationen (7), kann davon ausgegangen werden, dass viele AU-Fälle aufgrund operativer Eingriffe erfolgen.

\section{Schlussfolgerung}

Die Ergebnisse der Auswertung zeigen, dass die Diagnose Varizen der unteren Extremitäten u. a. aufgrund der Häufigkeit der AU auch in der Erwerbsbevölkerung einen bedeutenden gesundheitlichen sowie wirtschaftlichen Faktor darstellt. Obwohl Varizen keine schwerwiegende Erkrankung sind, besitzen sie eine hohe Public Health Relevanz. Ein Großteil der Bevölkerung erkrankt im Laufe des Lebens an Varizen, welche Beschwerden sowie wirtschaftliche Schäden bedingen können. Diese Auswertung erweitert das Wissen um aktuelle alters- und berufsgruppenabhängige Häufigkeiten einer Einzeldiagnose, die in dieser Art noch nicht berichtet wurden. Der Handlungsbedarf für Prävention (primär und sekundär) und Arbeitsgestaltung sowie der Forschungsbedarf wird damit unterstrichen.

Es ist davon auszugehen, dass bei den heute Jüngeren der bekannte Anstieg der AU aufgrund von HKE mit dem Alter bedingt durch die höhere Prävalenz der Risikofaktoren (Adipositas, Bewegungsarmut) eher stärker ausfällt (40). Varizen als direkte bzw. indirekte (z. B. Komplikationen wie Ulcus cruris) Ursache von AU steigen mit dem Alter stark an. Da in Zukunft wesentlich mehr ältere Erwerbstätige zu erwarten sind (41), soll versucht werden mit geeigneten primär und auch sekundär präventiven Maßnahmen einen Anstieg der AU-Fälle durch Varizen in der Erwerbsbevölkerung zu verhindern. Offen bleibt, in welchem Umfang betriebliche Maßnahmen mit anderen Zielsetzungen, wie z. B. die Reduktion der körperlichen Unterforderung am Arbeitsplatz durch Einführung und Nutzung von Steharbeitsplätzen, im Zusammenhang mit der Entstehung von Varizen der unteren Extremitäten, zu bewerten sind.

Varizen sind in manuellen Berufen der Produktion und in Dienstleistungsberufen, in denen auch Arbeiten im Stehen charakteristisch ist, überdurchschnittlich häufig ein Grund für AU. Berufs- spezifische Präventionsansätze, z. B. durch die Verminderung von langdauerndem Stehen oder Früherkennung im Rahmen der arbeitsmedizinischen Vorsorge, sollten hier teilweise schon früh etabliert werden. Diese Maßnahmen sollen nicht nur auf Verhaltensprävention, sondern auch auf die Verhältnisprävention zielen.

\section{Interessenkonflikt}

Die Autoren geben an, dass kein Interessenkonflikt besteht.

\section{Ethikkommission, Einverständniserklärung}

Die Konsultation einer Ethikkommission ist bei Analysen, die ausschließlich auf Sekundärdaten basieren, nicht erforderlich. (Laut: Gute Praxis Sekundärdatenanalyse (GPS), Leitlinien und Empfehlungen, 3. Fassung 2012, geringfügig modifiziert 2014)

Es wurden ausschließlich aggregierte Daten übermittelt und ausgewertet. Rückschlüsse/Bezüge zu einzelnen Personen sind nicht möglich. Daher sind keine Einverständniserklärungen erforderlich.

Literatur

[1] Gyberg V, Ryden L. Policymakers' perceptions of cardiovascular health in Europe. Eur J Cardiovasc Prev Rehabil 2011; 18 (5): 745-53

[2] Böhm K. Gesundheit und soziale Sicherung. In: Statistisches Bundesamt (Destatis), Wissenschaftszentrum Berlin für Sozialforschung (WZB), Hrsg. Datenreport 2016. 1. Auflage. Bonn, Bundeszentrale für politische Bildung (bpb). 2016: 273-339

[3] Robert Koch-Institut, Hrsg. Wie steht es um unsere Gesundheit. In: Gesundheit in Deutschland - Gesundheitsberichterstattung des Bundes. Berlin. H. Hennemann GmbH 2015: 18-144

[4] Statistisches Bundesamt (Destatis). Gesundheit: Krankheitskosten 2015 - Fachserie 12 (2017). Im Internet: https://www.destatis.de/DE/ Publikationen/Thematisch/Gesundheit/Krankheitskosten/KrankheitskostenJahr2120721159004.pdf?; Stand: 02.05.2018

[5] Zentralinstitut für die kassenärztliche Versorgung (ZI). Die 50 häufigsten ICD-10-Schlüsselnummern nach Fachgruppen Jahr 2015 (2016). Im Internet: https://www.zi.de/cms/fileadmin/images/content/PDFs_alle/ Die_50_häufigsten_ICD-2015.pdf; Stand: 02.05.2018

[6] Bundesministerium für Gesundheit. Arbeitsunfähigkeit: Fälle und Tage nach Diagnosen 2015 (2015). Im Internet: https://www.bundesgesundheitsministerium.de/fileadmin/Dateien/3_Downloads/Statistiken/ GKV/Geschaeftsergebnisse/AU_Faelle_nach_Diagnosen_2015.pdf; Stand: 02.05 .2018

[7] Nüllen H, Noppeney T. Sozialmedizinische und ökonomische Aspekte der Varikose. In: Noppeney T, Nüllen H, Hrsg. Varikose: Diagnostik Therapie - Begutachtung. 1. Auflage. Berlin: Springer 2010: 61-66

[8] Statistisches Bundesamt (Destatis). Gesundheit: Tiefgegliederte Diagnosedaten der Krankenhauspatientinnen und -patienten 2015 (2016). Im Internet: https://www.destatis.de/DE/Publikationen/Thematisch/Gesundheit/Krankenhaeuser/TiefgegliederteDiagnosedaten. html; Stand: 02.05.2018

[9] Rabe E, Pannier F. Epidemiologie chronischer Venen. In: Noppeney T, Nüllen H, Hrsg. Varikose: Diagnostik - Therapie - Begutachtung. 1. Auflage. Berlin: Springer 2010. 37-43 
[10] Dimakakos E, Syrigos K, Scliros E et al. Prevalence, risk and aggravating factors of chronic venous disease: an epidemiological survey of the general population of Greece. Phlebology 2013; 28 (4): 184-90

[11] Bihari I, Tornoci L, Bihari P. Epidemiological study on varicose veins in Budapest. Phlebology. 2012, 27;2: 77-81

[12] Fischer $\mathrm{H}$, Hrsg. Venenleiden: eine repräsentative Untersuchung in der Bevölkerung der Bundesrepublik Deutschland (Tübinger Studie). 1. Auflage. München: Urban \& Schwarzenberg 1981: XI

[13] Rentenzugänge wegen verminderter Erwerbsfähigkeit (Primärquelle: Deutsche Rentenversicherung Bund (DRV Bund), Statistik des Rentenzugangs (2016). Im Internet: www.gbe-bund.de (Stichwortrecherche: Rentenzugang Varizen); Stand: 02.05.2018

[14] Rabe E. Bonner Venenstudie der Deutschen Gesellschaft für Phlebologie. Phlebologie 2003; 32 (1) 1-14

[15] Brand FN, Dannenberg AL, Abbott RD et al. The epidemiology of varicose veins: the Framingham Study. Am J Prev Med 1988; 4 (2): 96-101

[16] Ebrahimi H, Amanpour F, Bolbol Haghighi N. Prevalence and risk factors of varicose veins among female hairdressers: a cross sectional study in north-east of Iran. J Res Health Sci 2015; 1 (2): 119-123

[17] Kroeger K, Ose C, Rudofsky G et al. Risk factors for varicose veins. Int Angiol 2004; 23 (1): 29-34

[18] Jawien $A$. The influence of environmental factors in chronic venous insufficiency. Angiology 2003; 54 (Suppl 1): 19-31

[19] Lee AJ, Evans C], Allan PL et al. Lifestyle factors and the risk of varicose veins: Edinburgh Vein Study. J Clin Epidemiol 2003; 56 (2): 171-179

[20] Carpentier PH, Maricq HR, Biro C et al. Risk factors, and clinical patterns of chronic venous disorders of lower limbs: a populationbased study in France. J Vasc Surg 2004: 40(4): 650-659

[21] Laurikka JO, Sisto T, Tarkka MR et al. Risk indicators for varicose veins in forty- to sixty-year-olds in the Tampere varicose vein study. World J Surg 2002; 26 (6): 648-651

[22] Sisto T, Reunanen A, Laurikka J et al. Prevalence and risk factors of varicose veins in lower extremities: mini-Finland health survey. Eur J Surg 1995; 161 (6): 405-414

[23] Reghunandanan R, Sood S, Reghunandanan V. Pulse wave velocity and lipid profile in varicose vein patients. Angiology 1995; 46 (5): 409-412

[24] Xu J, Shi GP. Vascular wall extracellular matrix proteins and vascular diseases. Biochim Biophys Acta 2014; 1842(11): 2106-2119

[25] Gomez I, Ozen G, Deschildre C et al. Reverse Regulatory Pathway (H2S/PGE2/MMP) in Human Aortic Aneurysm and Saphenous Vein Varicosity. PloS one 2016; 11 (6): e0158421. https://doi.org/10.1371/ journal.pone.0158421

[26] Bahk JW, Kim H, Jung-Choi K et al. Relationship between prolonged standing and symptoms of varicose veins and nocturnal leg cramps among women and men. Ergonomics 2012; 55(2): 133-139

[27] Tüchsen F, Hannerz H, Burr $\mathrm{H}$ et al. Prolonged standing at work and hospitalisation due to varicose veins: a 12 year prospective study of the Danish population. Occup Environ Med 2005; 62(12): 847-850
[28] Tabatabaeifar S, Frost P, Andersen JH et al. Varicose veins in the lower extremities in relation to occupational mechanical exposures: a longitudinal study. Occup Environ Med 2015; 72(0): 330-337

[29] Kontosic I, Vukelic M, Drescik I et al. Work conditions as risk factors for varicose veins of the lower extremities in certain professions of the working population of Rijeka. Acta Med Okayama 2000; 54 (1): 33-38

[30] Ziegler S, Eckhardt G, Stoger R et al. High prevalence of chronic venous disease in hospital employees. Wien Klin Wochenschr 2003; 115 (15-16): 575-579

[31] Wittig P, Nöllenheidt C, Brenscheidt S. Grundauswertung der BIBB/ BAuA-Erwerbstätigenbefragung mit Schwerpunkten Arbeitsbedingungen, Arbeitsbelastungen und gesundheitliche Beschwerden. 2012 (2013). Im Internet: https://www.baua.de/DE/Angebote/Publikationen/Berichte/Gd73.html; Stand: 02.05.2018

[32] Liebers F, Brendler C, Latza U. Berufsspezifisches Risiko für das Auftreten von Arbeitsunfähigkeit durch Muskel-Skelett-Erkrankungen und Krankheiten des Herz-Kreislauf-Systems: Bestimmung von Berufen mit hoher Relevanz für die Prävention. Dortmund: BAuA; 2016

[33] Graubner B, Deutsches Institut für Medizinische Dokumentation und Information (DIMDI). ICD-10-GM 2008, Systematisches Verzeichnis: Internationale statistische Klassifikation der Krankheiten und verwandter Gesundheitsprobleme, 10. Revision, German Modification, Version 2008. Köln: Dt. Ärzte-Verl; 2007

[34] Bundesanstalt für Arbeit (BA). Klassifizierung der Berufe - Systematisches und alphabetisches Verzeichnis der Berufsbenennungen. Nürnberg: Bundesanstalt für Arbeit (BA); 1988

[35] Blossfeld H-P. Bildungsexpansion und Berufschancen - empirische Analysen zur Lage der Berufsanfänger in der Bundesrepublik. Frankfurt: Campus; 1985

[36] Brendler C, Liebers F, Latza U. Berufsgruppen- und altersabhängige Unterschiede in der Arbeitsunfähigkeit durch häufige Herz-KreislaufErkrankungen am Beispiel der essenziellen Hypertonie und des akuten Myokardinfarktes. Bundesgesundheitsbl Gesundheitsforsch Gesundheitsschutz. 2013, 56;3: 381-390

[37] Schultz-Ehrenburg U, Reich-Schupke S, Robak-Pawelczyk B, Rudolph TM, C, Weindorf N, Hirche H, Altmeyer P, Stücker M. Prospective epidemiological study on the beginning of varicose veins. Phlebologie. 2009, 38;1: 17-25

[38] Liebers F, Brendler C, Latza U. Alters- und berufgruppenabhängige Unterschiede in der Arbeitsunfähigkeit durch häufige Muskel-SkelettErkrankungen. Bundesgesundheitsbl Gesundheitsforsch Gesundheitsschutz. 2013, 56;3: 367-380

[39] Latza U. Umgang mit Querschnittstudien in der Arbeitswelt. Zentralblatt Arbeitsmed. 2009;59: 6-10

[40] Kurth BM. Erste Ergebnisse aus der „Studie zur Gesundheit Erwachsener in Deutschland“ (DEGS). Bundesgesundheitsbl Gesundheitsforsch Gesundheitsschutz. 2012, 55: 1-11

[41] Nowossadek E. Demografische Alterung und Folgen für das Gesundheitswesen. Hrsg. Robert Koch-Institut Berlin. GBE kompakt. 2012, 3;2 